\title{
Strain distribution of repaired articular cartilage defects by tissue engineering under compression loading
}

\author{
Shilei Wang ${ }^{1}$, Yan Bao', Yinjie Guan², Chunqiu Zhang ${ }^{1,2^{*}} \mathbb{D}$, Haiying Liu', Xu Yang ${ }^{2}$, Lilan Gao ${ }^{1}$, Tongtong Guo ${ }^{3^{*}}$
} and Qian Chen ${ }^{2}$

\begin{abstract}
Background: It is difficult to repair cartilage damage when cartilage undergoes trauma or degeneration. Cartilage tissue engineering is an ideal treatment method to repair cartilage defects, but at present, there are still some uncertainties to be researched in cartilage tissue engineering including the mechanical properties of the repaired region.

Methods: In this study, using an agarose gel as artificial cartilage implanted into the cartilage defect and gluing the agarose gel to cartilage by using the medical bio-adhesive, the full-thickness and half-thickness defects models of articular cartilage in vitro repaired by tissue engineering were constructed. Strain behaviors of the repaired region were analyzed by the digital correlation technology under 5, 10,15, and 20\% compressive load.

Results: The axial normal strain (Ex) perpendicular to the surface of the cartilage and lateral normal strain (Ey) as well as shear strain (Exy) appeared obviously heterogeneous in the repaired region. In the full-defect model, Ex showed depthdependent strain profiles where maximum Ex occurs at the low middle zone while in the half-defect mode, Ex showed heterogeneous strain profiles where maximum Ex occurs at the near deep zone. Ey and Exy at the interface site of both models present significantly differed from the host cartilage site. Ey and Exy exhibited region-specific change at the host, interface, and artificial cartilage sites in the superficial, middle, and deep zones due to the artificial cartilage implantation.

Conclusion: Both defect models of cartilage exhibited a heterogeneous strain field due to the engineered cartilage tissue implant. The abnormal strain field can cause the cells within the repaired area to enter complex mechanical states which will affect the restoration of cartilage defects.
\end{abstract}

Keywords: Cartilage tissue engineering, Cartilage defects, Strain distribution, Digital image correlation

\section{Background}

Articular cartilage repair and regeneration continue to be largely intractable due to its poor regenerative properties [1]. Traditional methods like autografts, allografts, or microfracture have been clinically employed to treat articular cartilage defects, but there are still many shortcomings associated with these therapies [2]. Articular cartilage tissue engineering holds great promise to repair,

\footnotetext{
* Correspondence: zcqarticle@163.com; tongtong.guo@hit.edu.cn

${ }^{1}$ Tianjin Key Laboratory of the Design and Intelligent Control of the Advanced Mechatronical System, Tianjin University of Technology, Tianjin 300384, China

${ }^{3}$ Nature Science Department, Harbin Institute of Technology, Shenzhen Campus, Shenzhen 518055, China

Full list of author information is available at the end of the article
}

regenerate, and/or improve injured or diseased articular cartilage functionality without the shortcomings.

In the past decades, enormous progress has been made in the optimization of strategies for cartilage tissue engineering. Cartilage tissue engineering has been used in clinical treatment, but to date, there are still many issues that need to be researched [3, 4]. In fact, after construct implantation, new tissue and surrounding host cartilage may degenerate [5]. Cartilage repair often results in fibrocartilaginous tissue without the production of normal hyaline cartilage $[4,6]$. The integration between the host cartilage and repair tissue is not successful enough [7]. The exact mechanism of progressive degeneration has not been elucidated due to many causes, but from the 
aspect of cartilage mechanobiology, a mechanical role can be suggested in this process.

The mechanical environment has significant effects on the maintenance, growth, and development of cartilage $[8,9]$, and similarly, the mechanical state of repaired articular cartilage are also regulated by cartilage repair and regeneration by tissue engineering in diarthrodial joints. The morphology, ultrastructure, and composition of articular cartilage results from the functional adaptations to its mechanical condition $[1,9,10]$. Cartilage tissue is sensitive to its mechanical environment [8-10]. A variety of excessive mechanical loading protocols result in both matrix damage and cell death $[9,10]$, while not enough mechanical loading exercise also causes cartilage damage [11]. Even moderate increases in load may be expected to accelerate the rates of both mechanical fatigue and wear [8]. When the conditions of repaired articular cartilage deviate from the physiological mechanical environment, cartilage degeneration may occur. Focal damage to articular cartilage is common in arthroscopy patients and may contribute to progressive tissue degeneration by altering the local mechanical environment [1, 12-14]. Under compression loading, stress concentration occurred in areas of cartilage near the edges of a focal defect, and local strains are increased markedly in these regions. Elevated strain may reach levels that induce cell death and matrix damage, and thus, the elevated strain resulting from a focal defect may become injurious to cartilage and induce degeneration [14]. Finite element models of joints with focal defects also predict the increased strain in the tissue adjacent to a defect [15].

Although the mechanical environment on focal defects of articular cartilage has been predicted theoretically and analyzed experimentally in in vitro systems, few reports focus on what the mechanical condition is after repairing cartilage defects by cartilage tissue engineering [10]. In current engineered cartilaginous tissue, most tissue-engineered constructs show inferior biomechanical properties compared with native articular cartilage in many patients $[16,17]$. The biomechanical properties could cause local biomechanical environmental changes in the repaired region of the knee joint to damage the restoration similar to that of defects in articular cartilage. Finite element analysis showed that different strain phases occurred in the repaired region with a biphasic swelling model [18] and under rolling depression conditions. Some stresses in the tissue-engineered cartilage or host cartilage obviously deviated from normal physiological conditions of the knee [19, 20].

In this paper, we will establish both repaired models of full-thickness defects and half-thickness defects of articular cartilage. The mechanical characterization of a repaired articular cartilage region will be investigated in an experiment containing compressive load by digital image correlation technology.

\section{Methods}

\section{Sample preparation}

Eight-month-old pig knee joints were obtained from a local slaughterhouse. A fine saw was used to harvest a cartilage-bone block resembling a fan-shaped sheet (Fig. 1) by cutting along the perpendicular to the articular surface of the trochlea site. The full-thickness defect samples $(n=4)$ were made at the trochlea site by a curved knife, and the half-thickness defects samples $(n=4)$ also were obtained from the femoral groove. The cartilage defect sample is $4 \pm$ $0.1 \mathrm{~mm}$ in length, and the depth of cartilage is $2 \pm 0.14 \mathrm{~mm}$. The dimension of the full-defect is $4 \mathrm{~mm} \times 3 \mathrm{~mm} \times 2.0 \mathrm{~mm}$ respectively corresponding to the samples' length, defect width, and depth of cartilage. The dimensions of the half-thickness defect are $4 \mathrm{~mm} \times 3 \mathrm{~mm} \times 1.0 \mathrm{~mm}$, also respectively corresponding to the samples' length, defect width, depth of cartilage. Before conducting the experiment, both types of cartilage defect samples were kept moist in a culture dish with phosphate buffered saline (PBS).

The artificial cartilage was made of agarose gel at 1.8\% concentration. Using the curved knife, a block of agarose gel was processed into a block of artificial cartilage with the same dimensions of the cartilage defect within the cartilage-bone block. Since the defect was processed by the same curved knife, this ensured the same shape of defects in the same samples. The Young's modulus of the agarose gel is $20 \mathrm{kPa}$ within linear elastic $0-0.6$ strain and $50 \mathrm{kPa}$ in nonlinear elastic $0.6-0.8$ strain, which we obtained by mechanical testing. This modulus of $20 \mathrm{kPa}$ of the gel corresponds to the modulus of tissue-engineering cartilage which is not functionally cultured under mechanical conditions [21].

The cartilage defect was repaired similarly as in clinical practice. The artificial cartilage was transplanted into the cartilage defect sample with the defect interface glued by the medical biological glue named Compont Medical Adhesive (Beijing Suncon Medical Adhesive Co.Ltd) which has been used in clinical surgery. Figure 1 shows the repaired model of cartilage defect using the application of agarose gel.

\section{Compression experiment}

The compression tests of repaired cartilage defect sample were performed on a MTF-100 testing machine (MTF$100, \pm 100 \mathrm{~N}$, Center of Mechanical Experiment in Shanghai University, China) (Fig. 2). The speckle patterns on the repaired region were generated by iron oxide nanoparticles (Beijing Dk Nano technology Co., LTD). The nanoparticles are hard and black making them suitable for creating speckles and are embedded into the repaired tissue surface. This is so the clear identified images of cartilage can be captured by camera. The cartilage repaired region was compressed against a 


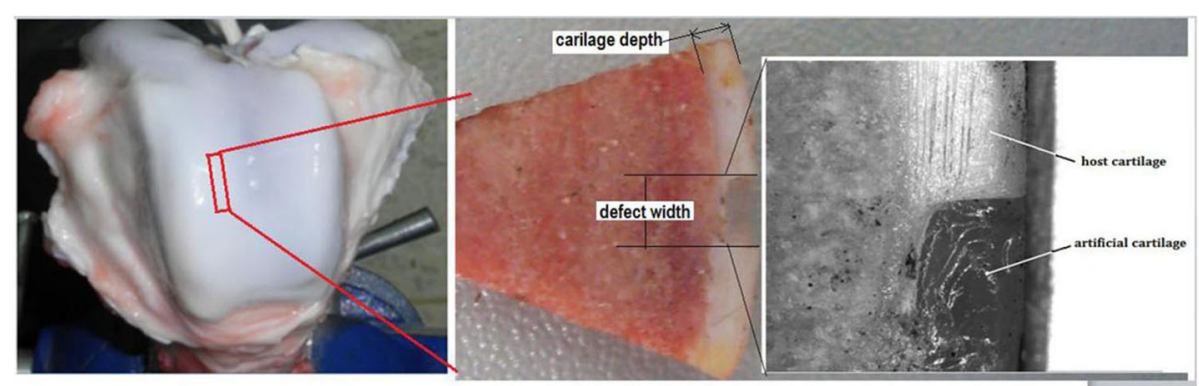

Fig. 1 Repaired model of cartilage defect by using agarose gel

transparent glass in the sample chamber during the compression experiment to avoid off-plane displacement from the repaired region surface to ensure the accuracy of strain calculations. The sample chamber was filled with PBS at room temperature (Fig. 2).

The subchondral bone was gripped and had exerted on it a tiny preload by the testing machine. Prior to any compressive loading, the initial thickness of each sample was measured microscopically. An image of the sample in its load-free reference state was first acquired. The samples were compressed at a speed of $1 \mathrm{~mm} / \mathrm{min}$ up to a $30 \%$ depth of cartilage at room temperature. During the loading process, samples are submerged in PBS, and interstitial fluid flowing from the compressed samples was dissolved into PBS, which ensures that clear images were captured by the camera. At the same time, the images of repaired cartilage were acquired at two frames per second during the entire testing process, and the images of the sample deformation were captured using the optimized DIC technique. The full-thickness defect samples and the half-thickness defects samples were loaded in the same conditions. The load was stopped when the apparent compression reached 30\%. There was

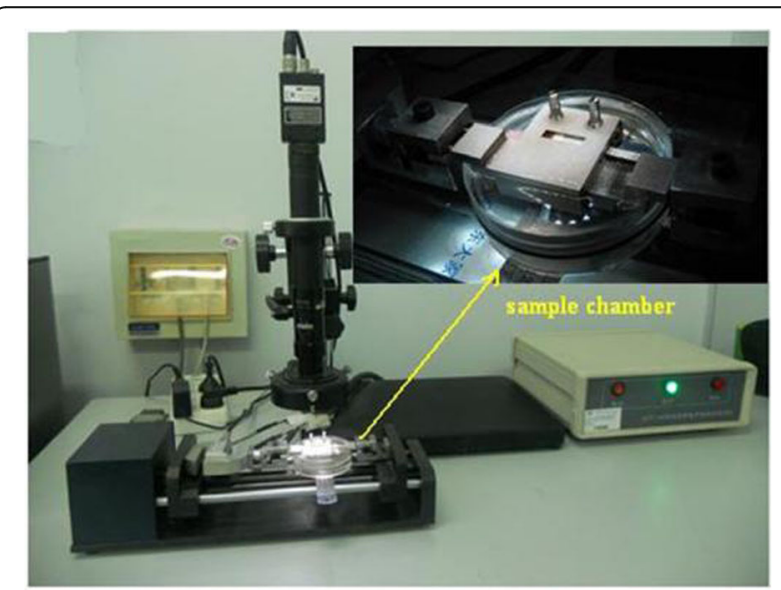

Fig. 2 Testing machine and camera system as well as sample chamber no cracking phenomenon at the interface of the repaired cartilage defect during the compression process.

\section{Strain testing location determination at feature site of repaired region}

Different locations of strain on the superficial, middle, and deep zones of the host, interface, and artificial tissue in repaired cartilage were researched. Locations of subregions are shown in Fig. 3: full-thickness defect sample including (1) representing the site of host cartilage (HC), (2) interface cartilage site (IC) between the host and artificial cartilage, and (3) artificial cartilage site (AC) in superficial zone(SD); (4) standing for $\mathrm{HC}$, (5) IC, and (6) $\mathrm{AC}$ in middle zone(MZ); and (7) standing for $\mathrm{HC}$, (8) $\mathrm{IC}$, and (9) AC in deep zone(DZ). There are certain differences of location in half-thickness defect sample (Fig. 3). Location 6 is near the interface site in the bottom of the artificial cartilage. Location 8 in the halfdefect sample is the host cartilage, while location 8 in the full-defect sample is in the artificial cartilage. Location 9 in the half-defect sample is the same in the host cartilage, while location 8 in the full-defect sample is same in the artificial cartilage.

The locations of sub-regions are arranged as follows: distance (D12) between location 1 and $2=1300 \mu \mathrm{m}$; D23/ D45/D56/D78/D89 = 1300 $\mu \mathrm{m} ; \quad \mathrm{D} 14 / \mathrm{D} 25 / \mathrm{D} 36=550 \mu \mathrm{m}$; D47/D58/D69 $=850 \mu \mathrm{m}$. The locations 3, 6, and 9 are on the center line or symmetric line of the defect region from the surface to the depth of cartilage. Distance between location 1 and the surface of cartilage is $140 \mu \mathrm{m}$.

The testing location strain is calculated using the average value on sub-regions of the circle area with a radius of 10 pixels $(\sim 40 \mu \mathrm{m})$. The strain at the feature site of the repaired region was respectively calculated at cartilage compression of 5, 10, 15, and 20\% (Fig. 4).

\section{Statistical analysis}

For the tests, a one-way analysis of variance (ANOVA) was carried out to determine the statistical variances among the strain values of host, interface, and artificial 


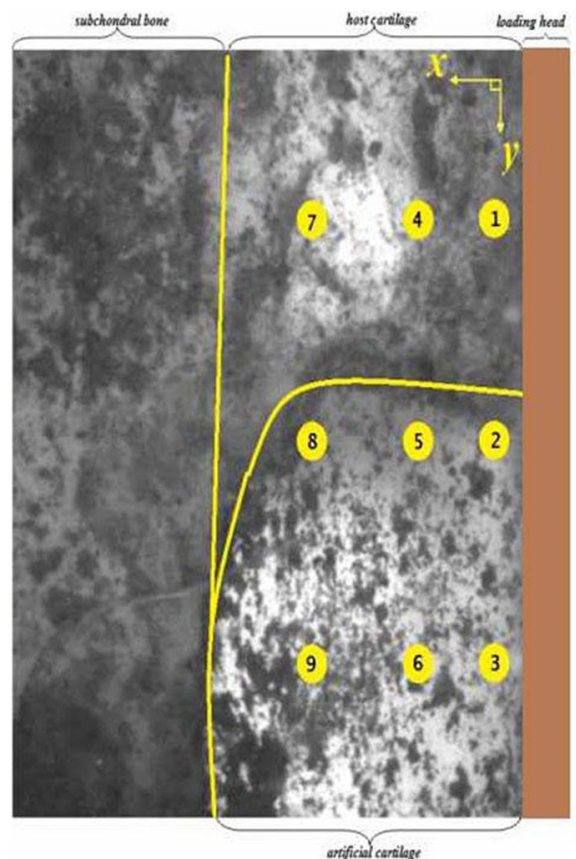

a full-thickness defect

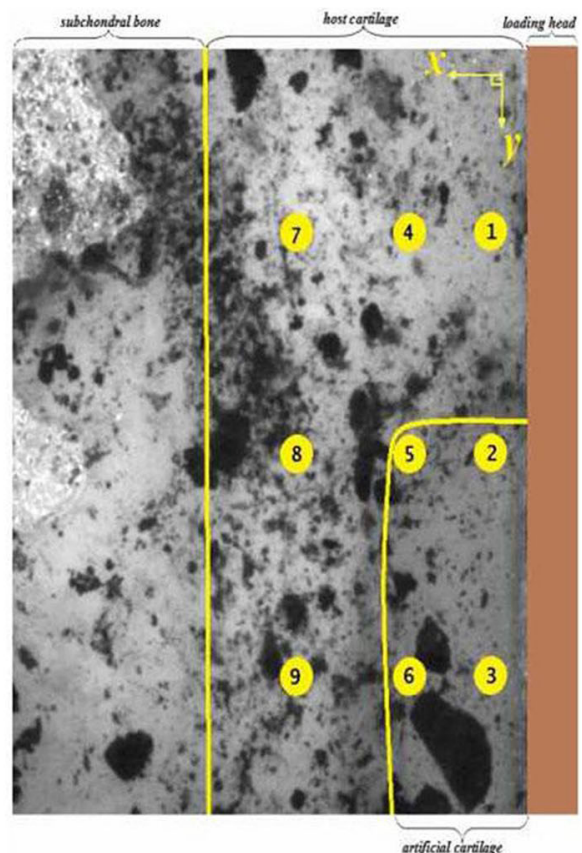

b half-thickness defect

Fig. 3 Cartilage defect samples. a: full-thickness defect; b: half-thickness defect. (Note: The yellow line shows the interface between artificial and host cartilage, and the right block part is a researched strain field). The location 1, 2, 3 represents the superficial zone of the host, interface and artificial tissue in repaired cartilage; The location 4, 5, 6 represents the middle zone of the host, interface and artificial tissue in repaired cartilage; The location 7, 8, 9 represents the deep zone of the host, interface and artificial tissue in repaired cartilage

cartilage zones and also in the superficial layer, middle, and deep zones of cartilage, respectively. A $p$ value of $<$ 0.05 was considered to be statistically significant. Test data used in the figures represented mean values, while the standard errors above and below mean values were indicated by error bars.

\section{Results}

Overall, the strain profiles in both full-thickness and half-thickness defect models of cartilage exhibited heterogeneous distribution through the host cartilage $(\mathrm{HC})$ to the interface(IC) and to artificial cartilage (AC).

\section{Full-thickness defects of cartilage models}

A representative strain distribution about axial normal strain $(\mathrm{E} x)$ and lateral normal strain (Ey) as well as shear strain (Exy) with an increase of compression to 5, 10, 15, $20 \%$ was shown in Fig. 5. Different strains at locations 1, 2, 3 standing for the host, interface, and artificial zone compared to repaired cartilage in superficial zone, 4, 5, 6 in the middle and 7, 8, 9 in the deep zone were shown in Fig. 6.

In the $\mathrm{X}$ direction (perpendicular to the cartilage surface), the $\mathrm{E} x$ of the repaired zone presents compressive strain distribution with characteristics of depth-dependent

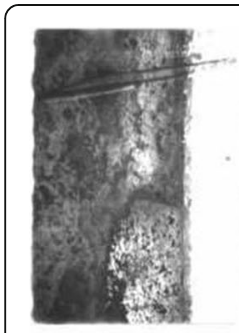

$5 \%$

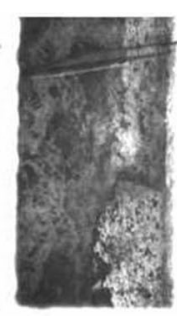

$10 \%$

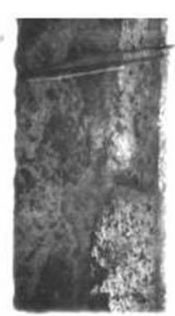

$15 \%$

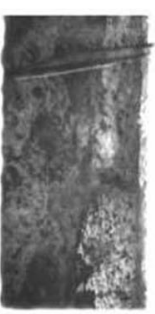

$20 \%$

a

full-thickness defect

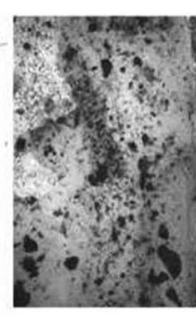

$5 \%$

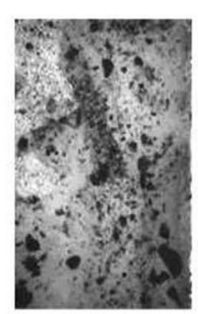

$10 \%$

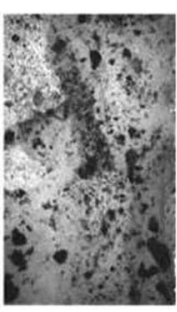

$15 \%$

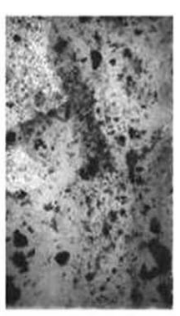

$20 \%$

b half-thickness defect

Fig. 4 Serial images of full-thickness defect and half-thickness defect at different 5, 10, 15, and 20\% compression 


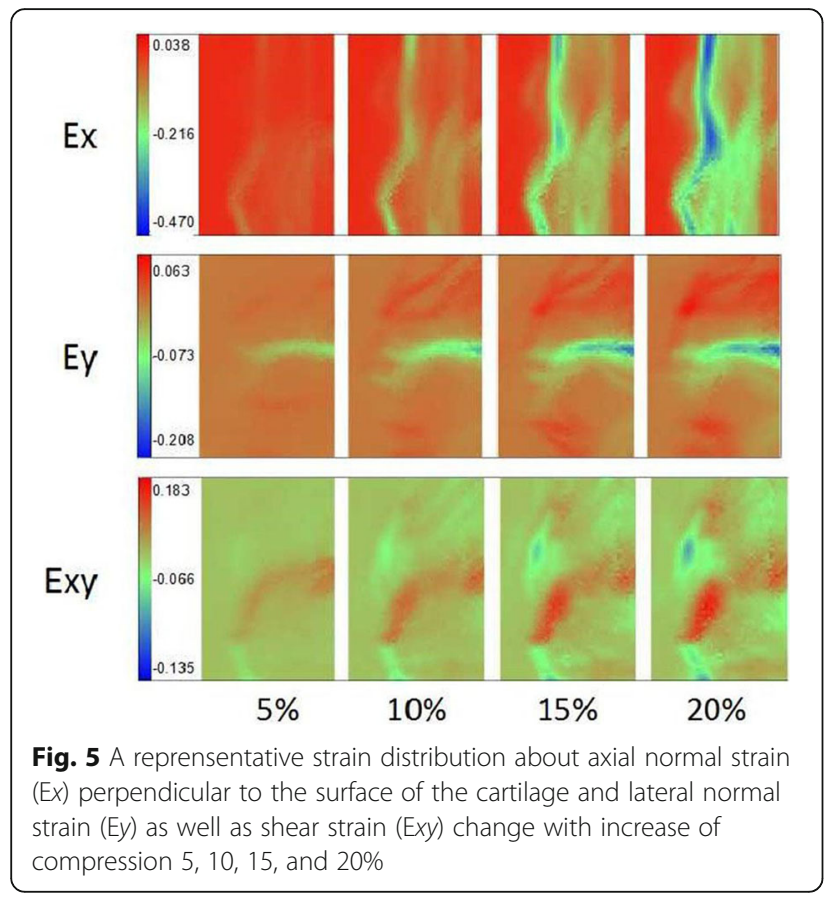

profiles during compression. The compressive strains decreased with depth in the strain profile, and the $\mathrm{E} x$ increased gradually with loading increase, which is similar to other researchers have found [13]. The largest Ex occurred at MZ about $1300 \mu \mathrm{m}$ from cartilage surface. The $\mathrm{E} x$ distribution at $\mathrm{AC}$ is higher than that at $\mathrm{HC}$ in $\mathrm{SZ}$ and is also more uniform. The $|\mathrm{E} x|$ of IC in DZ is larger than $\mathrm{AC}$ while lower than the one at $\mathrm{HC}$ in $\mathrm{DZ}$ during 5 , $10,15,20 \%$ compression. However, there are no significant differences in $\mathrm{E} x$ among $\mathrm{HC}$, IC, and $\mathrm{AC}(p=0.644)$.

In the $\mathrm{Y}$ direction (parallel to the surface of cartilage), the Ey of IC at SZ and MZ obviously occur compressive strain, and the $|\mathrm{E} y|$ of $\mathrm{SZ}$ is larger than MD and DZ, whereas the Ey of $\mathrm{HC}$ and $\mathrm{AC}$ appears to be tensile strain, though the Ey distribution is still depth-dependent like $\mathrm{E} x$ distribution. The $|\mathrm{E} y|$ of $\mathrm{HC}$ in $\mathrm{SZ}$ and $\mathrm{MZ}$ are larger than $\mathrm{AC}$. The Ey of IC at SZ increases rapidly at the start of compression and slowly at the end. At 20\% compression, the maximum $|\mathrm{E} y|$ of IC in SD is near four times as the one in $\mathrm{AC}$, and 3.5 times as the value in $\mathrm{HC}$. There is significantly different $\mathrm{E} y$ among $\mathrm{HC}, \mathrm{IC}$, and AC $(p=0.002)$.

As for the shear strain $(E x y)$ in the restored region, the Exy within the repaired zone appears to display regionspecific change at $\mathrm{HC}, \mathrm{IC}$, and AC. The Exy of IC within the repaired zone is significantly different from $\mathrm{HC}$ and $\mathrm{AC}(p=0.001)$, even the Exy directions of $\mathrm{AC}$ in $\mathrm{SZ}$ and of $\mathrm{HC}$ in $\mathrm{HC}$ changes with increases in compression. The maximum $|E x y|$ at IC of DZ is about four times the one at $\mathrm{AC}$ of $\mathrm{DZ}$ with repaired zone at $20 \%$ compression. The Exy of $\mathrm{HC}$ in DZ appears to go in the opposite direction of AC during the entire compression process.

\section{Half-thickness defects of cartilage models}

Like full-thickness defects, the half-thickness defects of cartilage have the same similar strain distribution at $\mathrm{HC}$, IC, and AC except local differences. The strain distribution indeed exhibited region-specific changes within the repaired zone. A representative strain distribution about $\mathrm{E} x, \mathrm{E} y$, and $\mathrm{Exy}$ with increase of compression to 5, 10, 15 , and $20 \%$ are shown in Fig. 7. Different strain at locations 1,2 , and 3 standing for the host, interface, and artificial zone compared to repaired cartilage in superficial zone, 4, 5, and 6 in the middle zone and 7, 8, and 9 in deep zones are shown in Fig. 8.

In the $\mathrm{X}$ direction (perpendicular to the cartilage surface), the $\mathrm{E} x$ distribution of $\mathrm{AC}$ is relatively more

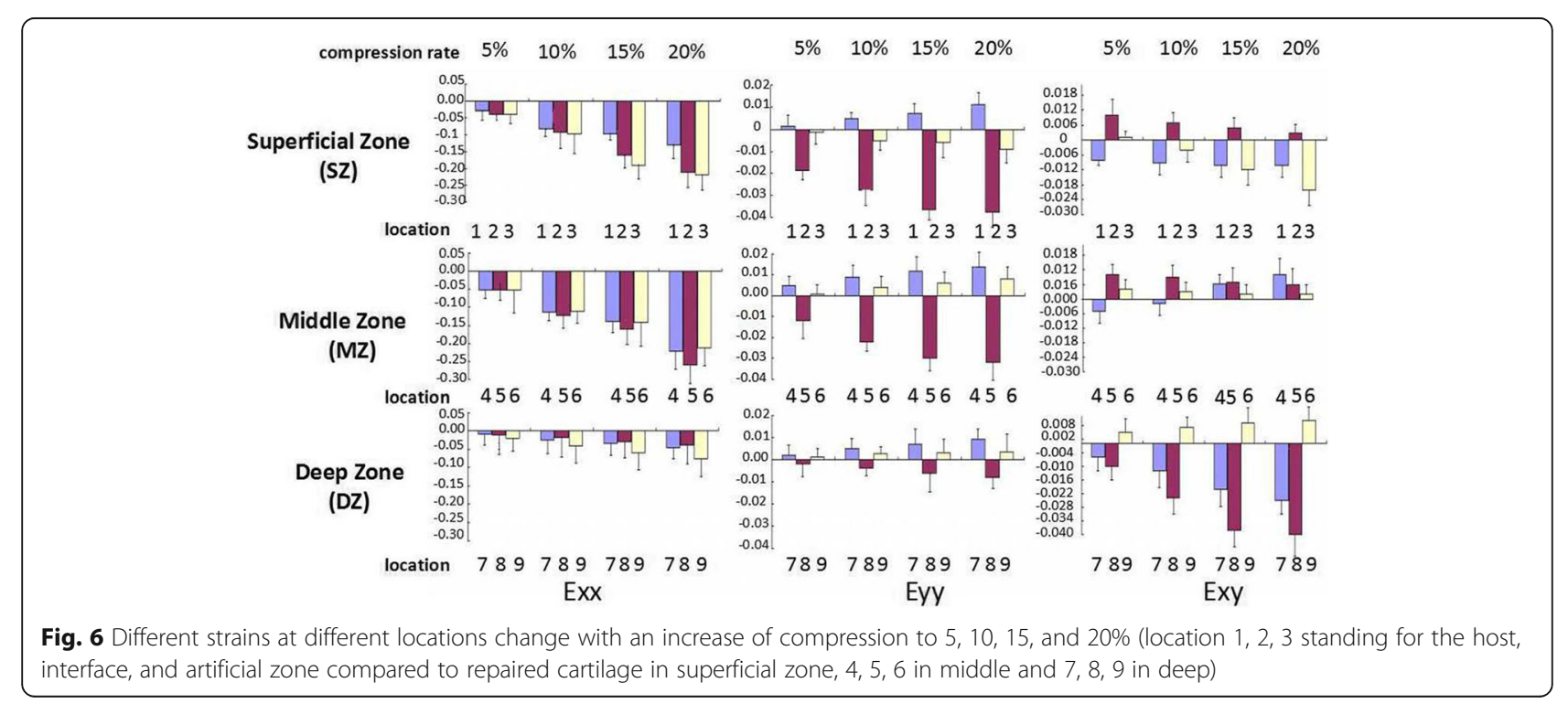




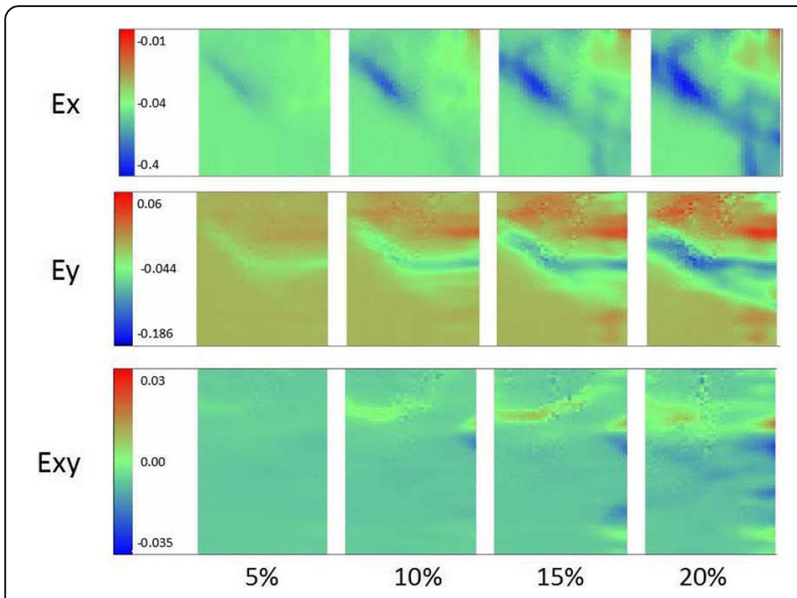

Fig. 7 A representative strain distribution about axial normal strain (Ex) perpendicular to the surface of the cartilage and lateral normal strain (Ey) as well as shear strain(Exy) changes with increase of compression to 5, 10, 15, and 20\%

uniform than that of HC. At $20 \%$ compression, the maximum $|\mathrm{E} x|$ of $\mathrm{HC}$ is 3.2 times that in $\mathrm{AC}$. There are significantly different $\mathrm{Ex}$ among $\mathrm{HC}, \mathrm{IC}$, and $\mathrm{AC}$ $(p=0.002)$. In the $\mathrm{Y}$ direction, the $\mathrm{E} y$ of IC mainly exhibits the compressive strain distribution similar to that in the full-thickness defect, while the $\mathrm{E} y$ of $\mathrm{HC}$ and $\mathrm{AC}$ appear to have tensile strain at $\mathrm{SD}$ and $\mathrm{MD}$. As for the $\mathrm{XY}$ direction shear strain, the direction and amount of $\mathrm{E} x y$ of $\mathrm{HC}$ is different from the one in IC and $\mathrm{AC}$ at the repaired region. These differences are obvious among the $\mathrm{HC}$, IC, and $\mathrm{AC}(p=0.002)$.

\section{Discussion}

According to the clinical surgery method of cartilage tissue engineering, we researched the mechanical characteristics of a repaired region after articular cartilage defects were transplanted by artificial cartilage. The mechanical characteristics of the repaired region only represent the time just after implantation. Cartilage development is affected by many factors, such as biological, chemical, material, and biophysical. Here, the research only applies to biophysical force effects. In fact, a comprehensive study should be performed at different time periods, which will be our future goal.

The strain in the restored area of cartilage appears to have complex variation, and it is related to many factors in the restored area such as the interface bonding, the shape of the defect, material mechanical properties, and the loading condition. In this study, the defect interface was adhered by biological glue, which has been used in clinical surgery, in order to ensure the experiment operated in a realistic surgical environment. There was no cracking phenomenon at the defect interface during the apparent compression process. We will further research the cause of cracking at the defect interface being caused by incomplete bonding and stress concentration at the interface. For the shape of defect, the defect is classified as a full-thickness defect model and half-thickness defect model of cartilage, and both defect interfaces appear to possess obvious stress concentration. Moreover, the strain distribution in the restored area is also related to the size of artificial cartilage transplanted into the defect area. The different shapes of the defects are also lack of researches. The material mechanical properties of artificial cartilage play an important role in strain profiles in the repaired region. If the agarose gel is incompressible, implanted artificial cartilage will cause complex changes of Ex and Exy. The strain in the restored area of cartilage indeed appears to have complex variation. The complex $\mathrm{E} x$ and Exy in this study may result from the artificial

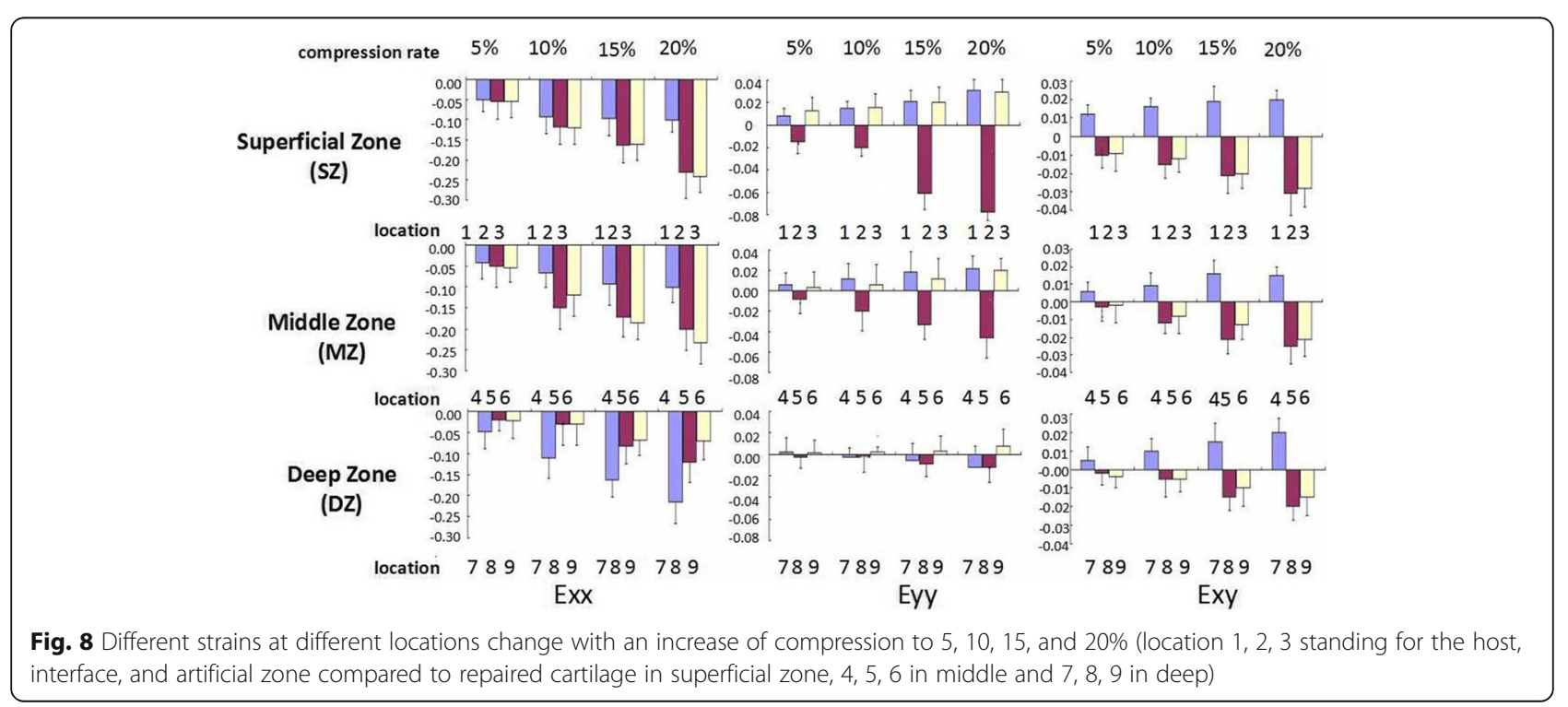


cartilage being incompressible. Compression as a loading force was applied in the experiment, and this kind of load is only typical of what is usually imposed on knee cartilage. In practice, articular cartilage is also affected by a rolling load and a sliding load, so it is essential to study the mechanical characteristics of the restored area of cartilage under these loads.

In the previous experimental cases, some scholars have established a finite element model of defect cartilage restoration to simulate and analyze the strain distribution at the restored area of host cartilage and artificial cartilage. This allowed them to explore the elastic modules, the amount of compression, and the size of defect area, and this affects the stress distribution at the restored area $[20,22]$. The results show that the amount of compression obviously affects the strain distribution at the restored area. For example, when the elastic module of artificial cartilage is $0.6 \mathrm{MPa}$, the difference of strain between the artificial cartilage and host cartilage with compression loading ranging from 5 to $30 \%$ is up to 3.26 times. The size of the defect area is also related to the strain distribution of cartilage, and it is also consistent with the previous experimental conclusions. In this experiment, the variation trend of strain of host cartilage away from the restored area decreased gradually from superficial layer to deep layer. The strain field showed in this experiment is consistent with Ahmed, Gao, and Zhang's findings who researched the strain distribution of intact cartilage respectively [23, 24].

In this experiment, a phenomenon was discovered in which the mechanical properties of artificial cartilage should be consistent with host cartilage, or otherwise, there will be residual stress after loading. When the compressive load is removed, the recovery timetable in the restored area of artificial cartilage and host cartilage appears to have obvious differences. The artificial cartilage recovered faster, whereas the host cartilage recovered slower because of its different viscoelastic properties. When the compression is at $30 \%$, the host cartilage required $30 \mathrm{~min}$ to be restored to its original height. And that the defect interface appears to contain residual stress, and this will affect the cartilage restoration. This area of research will be looked at in the future.

In this study, the artificial cartilage will help to reveal one of the mechanical states in restoration of full-thickness and half-thickness defects of cartilage in only one kind of elastic modulus. At present, when the loading rate range is from 0.038 to $4.05 \%$ per second, the elastic modulus of artificial cartilage ranges from 0.94 to $3.035 \mathrm{Mpa}$ [24]. The elastic modulus of agarose used in this experiment is about 20000pa, which is still lower than the native cartilage, but it is consistent with the mechanical properties of tissueengineered cartilage at present. If the mechanical properties of the restored area need to be clearly researched after artificial cartilage is implanted into the defect cartilage, it is essential to use different concentrations of agarose to make a higher gradient elastic modulus to research. The agarose gel with an elastic modulus equal to or higher than cartilage is also needed to compare to each other. The carrageenan may be jointed to enhance the toughness and elastic modulus for researching the mechanical properties of the restored area of cartilage in the future.

\section{Conclusion}

In the article, we simulated the artificial cartilage and host cartilage that is completely bonded, and is not completely bonded clinically, and its real boundary conditions are more complex, so that there are some differences between the simulation results and the actual situation. In addition, the agarose appears to have a higher brittleness in the experiment, which is different from the natural cartilage. On the other hand, pig knee joints and human knee have substantial differences in geometry and loads, but these experiments also have some theoretical and reference value; they reveal the mechanical behaviors of defect articular cartilage in a restored area with a compressive load. It will help to improve the mechanical properties of artificial cartilage implants and explore the suitable mechanical function indicators of artificial cartilage in implanting and restoration during cartilage tissue engineering in the future.

\section{Abbreviations \\ Ex: Axial normal strain perpendicular to the surface of the cartilage; Exy: Shear strain; Ey: Lateral normal strain}

\section{Acknowledgements}

Not applicable

\section{Funding}

National Natural Science Key Foundation of China (Nos.11432016), National Natural Science Foundation of China (Nos.11672208, 11572222, and 11402172)

\section{Availability of data and materials}

Data and materials presented in this paper can be shared upon request.

\section{Authors' contributions}

$\mathrm{CZ}$, main operator, Ph.D. is in charge of giving the main idea and the whole design. SW and YB are in charge of experimental design and implementation. All authors are in charge of (1) the acquisition of data and data analysis and image processing, (2) drafting the manuscript or revising it critically for important intellectual content, (3) and giving the final approval of the version to be published. Each author has participated sufficiently in the work to take public responsibility for appropriate portions of the content. All authors read and approved the final manuscript.

\section{Ethics approval and consent to participate}

There are no ethics problems in our experiments and article.

Consent for publication

Not applicable

Competing interests

The authors declare that they have no competing interests. 


\section{Publisher's Note}

Springer Nature remains neutral with regard to jurisdictional claims in published maps and institutional affiliations.

\begin{abstract}
Author details
${ }^{1}$ Tianjin Key Laboratory of the Design and Intelligent Control of the Advanced Mechatronical System, Tianjin University of Technology, Tianjin 300384, China. ${ }^{2}$ Cell and Molecular Biology Laboratory, Department of Orthopaedics, Alpert Medical School of Brown University/Rhode Island Hospital, 1 Hoppin St., Ste. 402, Providence, RI 02903, USA. ${ }^{3}$ Nature Science Department, Harbin Institute of Technology, Shenzhen Campus, Shenzhen 518055, China
\end{abstract}

Received: 1 December 2017 Accepted: 19 January 2018

Published online: 30 January 2018

\section{References}

1. Venäläinen MS, Mononen ME, Salo J, et al. Quantitative evaluation of the mechanical risks caused by focal cartilage defects in the knee. Sci Rep. 2016; 6:37538.

2. Caldwell KL, Wang J. Cell-based articular cartilage repair: the link between development and regeneration. Osteoarthr Cartil. 2015;23(3):351.

3. Kock L, van Donkelaar CC, Ito K. Tissue engineering of functional articular cartilage: the current status. Cell Tissue Res. 2012;347(3):613-27.

4. Kwon $\mathrm{H}$, Paschos NK, Hu JC, et al. Articular cartilage tissue engineering: the role of signaling molecules. Cell Mol Life Sci. 2016;73(6):1-22.

5. Kim BS, Park IK, Hoshiba $T$, et al. Design of artificial extracellular matrices for tissue engineering. Prog Polym Sci. 2011;36(2):238-68.

6. Becerra J, Andrades JA, Guerado E, et al. Articular cartilage: structure and regeneration. Tissue Eng Part B Rev. 2010;16(6):617-27.

7. Farr J, Cole B, Dhawan A, et al. Clinical cartilage restoration: evolution and overview. Clin Orthop Relat Res. 2011:469(10):2696-705.

8. Wong M, Carter DR. Articular cartilage functional histomorphology and mechanobiology: a research perspective. Bone. 2003;33(1):1

9. Li K, Zhang C, Qiu L, Gao L, Zhang X. Advances in application of mechanical stimuli in bioreactors for cartilage tissue engineering. Tissue Eng Part B Rev. 2017; https://doi.org/10.1089/ten.TEB.2016.0427.

10. Guilak F, Butler DL, Goldstein SA, et al. Biomechanics and mechanobiology in functional tissue engineering. J Biomech. 2014;47(9):1933-40.

11. Julkunen P, Halmesmäki EP, livarinen J, Rieppo L. Effects of growth and exercise on composition, structural maturation and appearance of osteoarthritis in articular cartilage of hamsters. J Anat. 2010;217(3):262-74.

12. Duda GN, Maldonado ZM, Klein P, et al. On the influence of mechanical conditions in osteochondral defect healing. J Biomech. 2005;38(4):843-51.

13. Wong BL, Sah RL. Effect of a focal articular defect on cartilage deformation during patello-femoral articulation. J Orthop Res. 2010;28(12):1554-61.

14. Gratz KR, Wong BL, Bae WC, et al. The effects of focal articular defects on cartilage contact mechanics. J Orthop Res. 2009;27(5):584-92.

15. Vahdati A, Wagner DR. Finite element study of a tissue-engineered cartilage transplant in human tibiofemoral joint. Comput Methods Biomech Biomed Engin. 2012;15(11):1211-21.

16. Huang AH, Farrell MJ, Mauck RL. Mechanics and mechanobiology of mesenchymal stem cell-based engineered cartilage. J Biomech. 2010;43(1): 128-36.

17. Zhang L, Hu J, Athanasiou KA. The role of tissue engineering in articular cartilage repair and regeneration. Crit Rev Biomed Eng. 2009:37:1-57.

18. Sztefek P, Vanleene M, Olsson R. Using digital image correlation to determine bone surface strains during loading and after adaptation of the mouse tibia. J Biomech. 2010;43(4):599.

19. Wentzell S, Sterling NR, Macione J, et al. Measuring strain using digital image correlation of second harmonic generation images. J Biomech. 2013; 46(12):2032-8

20. Shu Qing Z, et al. Mechanical state researches on repairing articular cartilage defects by tissue engineering. J Clin Rehabil Tissue Eng Res. 2011; 15(20):253-8.

21. Hung $C T$, Mauck RL, Wang $C B$, et al. Erratum: a paradigm for functional tissue engineering of articular cartilage via applied physiologic deformational loading. Ann Biomed Eng. 2004;32(1):35-49.
22. Zhang $\mathrm{S}$, et al. The simulation of mechanical states of repaired Articular cartilage. International conference on bioinformatics and biomedical engineering IEEE; 2010. p. 1-4.

23. Ahmed TA, Hincke MT. Strategies for articular cartilage lesion repair and functional restoration. Tissue Eng Part B Rev. 2010;16(3):305-29.

24. Gao LL, Zhang CQ, Dong LM, et al. Description of depth-dependent nonlinear viscoelastic behavior for articular cartilage in unconfined compression. Mater Sci Eng C. 2012;32(2):119-25.

\section{Submit your next manuscript to BioMed Central and we will help you at every step:}

- We accept pre-submission inquiries

- Our selector tool helps you to find the most relevant journal

- We provide round the clock customer support

- Convenient online submission

- Thorough peer review

- Inclusion in PubMed and all major indexing services

- Maximum visibility for your research

Submit your manuscript at www.biomedcentral.com/submit 